$1-2012$

\title{
A New Algorithm for Detecting Central Apnea in Neonates
}

Hoshik Lee

William \& Mary, hoshik.lee@wm.edu

Craig G. Rusin

Douglas E. Lake

William \& Mary

et al.

John B. Delos

William \& Mary, jbdelos@wm.edu

\section{Recommended Citation}

Lee, Hoshik; Rusin, Craig G.; Lake, Douglas E.; al., et; and Delos, John B., A New Algorithm for Detecting Central Apnea in Neonates (2012). Physiological Measurement, 33(1), 1-17.

https://doi.org/10.1088/0967-3334/33/1/1

This Article is brought to you for free and open access by the Arts and Sciences at W\&M ScholarWorks. It has been accepted for inclusion in Arts \& Sciences Articles by an authorized administrator of W\&M ScholarWorks. For more information, please contact scholarworks@wm.edu. 


\title{
A new algorithm for detecting central apnea in neonates
}

\author{
Hoshik Lee ${ }^{1}$, Craig G Rusin ${ }^{2}$, Douglas E Lake ${ }^{2,3}$, Matthew T Clark ${ }^{4}$, \\ Lauren Guin $^{2}$, Terri J Smoot ${ }^{2}$, Alix O Paget-Brown ${ }^{5}$, \\ Brooke D Vergales $^{5}$, John Kattwinkel ${ }^{5}$, J Randall Moorman ${ }^{2}$ \\ and John B Delos ${ }^{1,6}$ \\ ${ }^{1}$ Department of Physics, College of William and Mary, Williamsburg, VA 23187, USA \\ 2 Department of Medicine, Division of Cardiovascular Medicine, University of Virginia Health \\ System, Charlottesville, VA 22908, USA \\ 3 Department of Statistics, University of Virginia, Charlottesville, VA 22904, USA \\ ${ }^{4}$ Department of Chemical Engineering, University of Virginia, Charlottesville, VA 22904, USA \\ ${ }^{5}$ Department of Pediatrics, Division of Neonatology, University of Virginia Health System, \\ Charlottesville, VA 22908, USA \\ E-mail: jbdelo@wm.edu
}

Received 19 September 2011, accepted for publication 15 November 2011 Published 7 December 2011

Online at stacks.iop.org/PM/33/1

\begin{abstract}
Apnea of prematurity is an important and common clinical problem, and is often the rate-limiting process in NICU discharge. Accurate detection of episodes of clinically important neonatal apnea using existing chest impedance (CI) monitoring is a clinical imperative. The technique relies on changes in impedance as the lungs fill with air, a high impedance substance. A potential confounder, however, is blood coursing through the heart. Thus, the cardiac signal during apnea might be mistaken for breathing. We report here a new filter to remove the cardiac signal from the CI that employs a novel resampling technique optimally suited to remove the heart rate signal, allowing improved apnea detection. We also develop an apnea detection method that employs the CI after cardiac filtering. The method has been applied to a large database of physiological signals, and we prove that, compared to the presently used monitors, the new method gives substantial improvement in apnea detection.
\end{abstract}

Keywords: apnea detection, cardiac artifact, chest impedance measurement (Some figures may appear in colour only in the online journal)

6 Author to whom any correspondence should be addressed. 
Abbreviations

$\begin{array}{ll}\text { ABD } & \text { Apnea associated with Bradycardia and oxygen Desaturation } \\ \text { CI } & \text { Chest Impedance } \\ \text { CI-CAR } & \text { Chest Impedance-Cardiac Artifact Removed } \\ \text { FCI } & \text { Filtered Chest Impedance } \\ \text { NICU } & \text { Neonatal Intensive Care Unit } \\ \text { VLBW } & \text { Very Low Birth Weight }(<1.5 \mathrm{Kg}) \\ \text { WAD } & \text { Weighted Apnea Duration }\end{array}$

\section{Introduction}

Apnea is very common in premature infants (Finer et al 2006). The breathing of all infants is irregular, and they all stop breathing for a few seconds at a time. Apnea is considered to be a clinical event if (Finer et al 2006) (A) cessation of breathing lasts for more than $20 \mathrm{~s}$, or (B) cessation of breathing lasts for more than $10 \mathrm{~s}$, and is accompanied by either bradycardia (slowing of the heart below 100 beats per minute) or oxygen desaturation $\left(\mathrm{SpO}_{2}<80 \%\right)$. In any such case, apnea is a serious clinical event that needs medical attention within seconds.

Clinical apneas occur in more than half of babies whose birth weight is less than $1500 \mathrm{~g}$, and in almost all infants whose birth weight is less than $1000 \mathrm{~g}$. Apnea may be a cause or an effect of many other clinical problems, such as hypoxemia, hypoglycemia, neurological injury or sepsis. Apnea is also a common manifestation of an immature neurological system in babies who were born very preterm, but otherwise have no clinical pathology. Three types of apnea are common in premature infants: obstructive apnea, central apnea and mixed apnea. Obstructive apnea is a blockage of the airway, typically accompanied by struggling or thrashing movements of the infant. Central apnea is cessation of respiratory drive, and the infant makes no effort to breathe. Mixed apneas typically begin with an obstructive event, and then change to central apnea. On the other hand, there is evidence that central apnea can also become obstructive apnea, so Poets (2010) suggests that the distinction between the two types should not be held too rigidly. When accompanied by bradycardia and/or oxygen desaturation, these apneas are serious clinical events that need immediate medical attention and investigation for associated pathology. Moreover, central apnea is taken to indicate immaturity of control of respiration, and discharge from our NICU is delayed until apneas have been absent for eight days (Darnall et al 1997).

Bedside monitors employ algorithms to detect apnea based on continuous monitoring of chest impedance (CI). Using electrodes that are also used to monitor the electrocardiogram (EKG), a small high-frequency (e.g. $52.6 \mathrm{KHz}$ ) voltage is applied to the chest, and the resulting high-frequency current is measured. The measured impedance is the applied voltage divided by observed current. The basic impedance is of the order of a few hundred ohms; it is related to the conductivity of muscle, skin, other tissues, and the contacts between electrodes and skin. When the infant is breathing, the impedance fluctuates by a few ohms in each breath. Air has low electrical conductivity, and more air in the lungs gives higher CI (Jong 2000). The beating of the heart also gives fluctuations of impedance, usually on the order of half an ohm. Blood has a high conductivity; as the heart empties and fills, the impedance rises and falls.

The heart rate slows during severe apnea, and there is increased time for diastolic filling and thus increased intracardiac volumes. As a result, CI changes due to cardiac activity are increased, and stand out all the more because the respiratory changes are absent. These increasingly large impedance fluctuations due to cardiac filling can reach thresholds for 

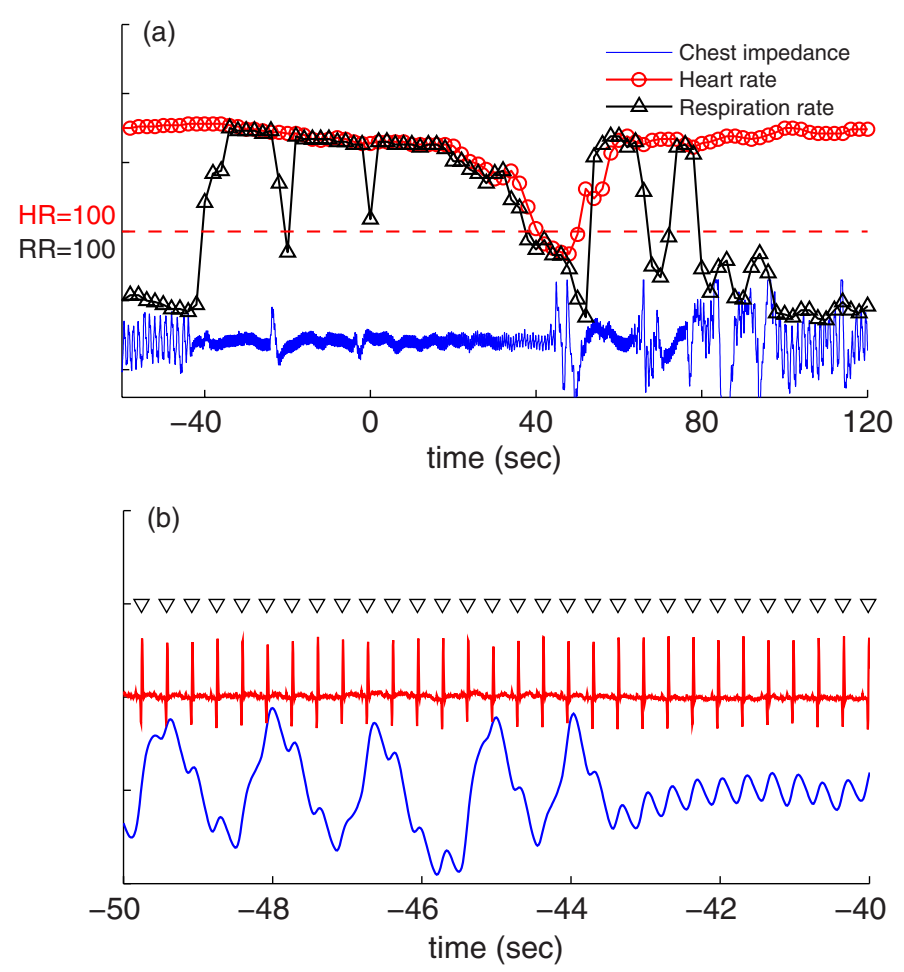

Figure 1. Apnea not detected by the monitor. (a) Chest impedance (blue, CI), heart rate (red) and respiration rate (black) signals from the bedside monitor. At $t=-43 \mathrm{~s}$, the CI changes, and the detected respiratory rate rises to match the heart rate. (b) EKG and CI expanded. During the apnea event starting at $t=-43 \mathrm{~s}$, the fluctuations in CI precisely match the EKG, indicating that they are due to the heart.

detection as breaths, foiling the monitor's apnea alarm. As a result, some episodes of central neonatal apnea are missed altogether, and some of those episodes are severe (figure 1).

We have systematically investigated this phenomenon of missed apneas in a large data set of waveforms and vital signs from the University of Virginia NICU. Finding it a common problem, we devised a new filtering strategy to remove the cardiac signal from the $\mathrm{CI}$, allowing more accurate apnea detection. The purpose of this paper is to report a new algorithm for detection and characterization of central apneas.

\section{Methods}

\subsection{Patient population}

We studied all admissions to the 45-bed University of Virginia NICU, a tertiary care referral center with more than 500 admissions yearly, almost one-fourth of them of very low birth weight $(<1500 \mathrm{~g}, \mathrm{VLBW})$. For development of methods to filter the cardiac component of the CI signal, we focused first on five VLBW infants with frequent apneas noted on the nursing apnea and bradycardia bedside flow sheets. 

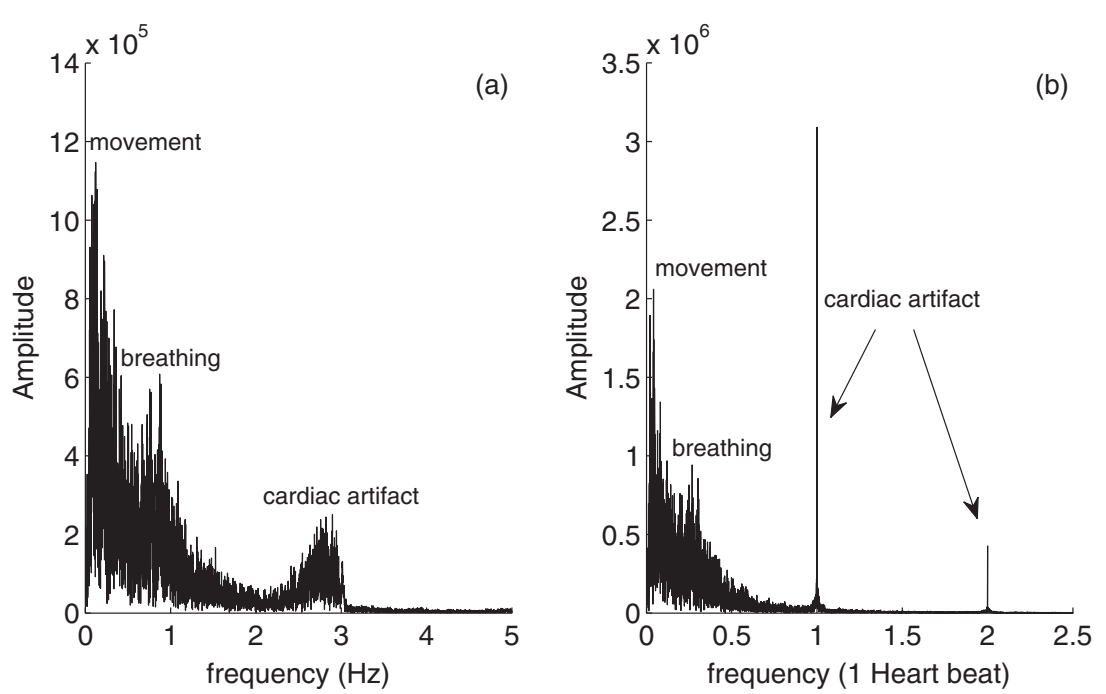

Figure 2. (a) Fourier transform of CI during an apnea event. We see low and broad peaks around $1 \mathrm{~Hz}$ and around $3 \mathrm{~Hz}$, but we cannot use this to filter the heart signal from the respiratory signal. (b) Fourier transform of CI using the heart as the clock. The cardiac artifact is now a set of narrow, sharp peaks at integer frequencies.

\subsection{Data acquisition, storage and analysis}

Our NICU uses GE monitors (models Solar 8000M and I, and Dash 3000, GE Healthcare, Milwaukee) to record three EKG leads (sampled at $240 \mathrm{~Hz}), \mathrm{CI}(60 \mathrm{~Hz})$, and oxygen saturation waveform $(120 \mathrm{~Hz})$, and average heart rate, respiration rate and $\mathrm{O}_{2}$ saturation $(0.5 \mathrm{~Hz})$, as well as monitor alarms. We have collected these data since January 2009 using the BedMaster (Excel Medical, Jupiter, FL) system and our parallel storage and computing cluster.

Data files are distributed equally among ten desktop personal computers having a total of 80 processing cores, 100 TB of hard disk storage and 30 gigabytes of memory. We developed a Matlab-based graphical interface for inspecting data, and for developing and testing numerical data analysis algorithms. Clinical data, such as respiratory support, demographics and apneabradycardia documentation, are collected from electronic medical records and the bedside flowsheets. We enter these data into a relational clinical database through a custom web interface.

\subsection{Cardiac artifact filter}

A number of methods have been proposed for eliminating the cardiac artifact from the CI signal based on the different frequencies of breaths and heartbeats (Wright and Triebel 1988, Judell 1983). However, extended apneas often lead to bradycardia, so the heart rate drops below $100 \mathrm{bpm}$, and may be well into the respiration frequency band. Therefore, a Fourier spectrum of the CI signal can be broadband and fail to distinguish cardiac and respiratory events (figure 2(a)).

To more effectively filter the cardiac component of CI, we use the heart itself as the clock (Wright and Triebel 1988); i.e. we digitally stretch or contract the time scale such that RR intervals are equally spaced, and the unit of time is thus one RR interval instead of $1 \mathrm{~s}$. The CI is resampled with an equal number of steps in each RR interval, so all peaks 
in the CI that are caused by heartbeats have equal spacing of one unit. Now the Fourier transform of the resampled CI has sharp narrow peaks at integer frequencies (figure 2(b)). The very low frequency content, moreover, is due to movement of the infant, or other unwanted baseline fluctuations; in any case, it does not represent regular neonatal respiration. The inverse transform after excluding heartbeat frequency and irrelevant very low frequencies is influenced neither by phasic blood flow through the heart nor by low frequency drift, and can be considered a more accurate measure of air flow in and out of the chest.

Details of the filter are given in the appendix. As discussed below, the method was validated through examination of hundreds of apnea episodes by three clinical experts.

\section{Results}

\subsection{An example of the analysis}

Figure 1(a) shows 3 min of bedside monitoring data during a prolonged episode of central neonatal apnea during which the apnea alarm did not sound. Initially the infant was breathing regularly at a rate of about 50 breaths per minute. Near $t=-43 \mathrm{~s}$, the CI signal (blue) falls in amplitude and rises in rate. Figure 1(b) is an expanded view, showing that the oscillations in CI synchronize with the EKG, establishing the source of the 180 per minute fluctuations as the beating of the heart. Over the next 60 to $80 \mathrm{~s}$, the oscillations in CI continue to be accurately synchronized with the EKG as the heart slows, and the detected heart and breathing rates fall together. Since the reported respiratory rate was always high, apnea was never detected by the monitor.

Figure 2 shows frequency-domain approaches to filtering the cardiac component of the CI signal. Figure 2(a) shows the Fourier spectrum of the CI signal. The bands around 1 and $3 \mathrm{~Hz}$ represent respiration and heart beats, respectively. The slowing heart rate, though, makes its peak broad and not suitable for filtering. Figure 2(b) shows the effect of resampling the CI in equally spaced increments of RR intervals. The periodic cardiac signal is now captured in narrow, sharp peaks at integer frequencies.

Figure 3 shows the filtered CI signal on compressed and expanded scales. Removal of the impedance changes caused by heartbeats reveals the nearly complete absence of chest movement due to breathing, and confirms the presence of central neonatal apnea. Figure 3(b) also shows the final step of the analysis, assignment of a probability of apnea using methods detailed in the appendix. The red bar (between $t=-43$ and $t=42$ ) in figure 3(b) indicates the detected 'ABD' event - apnea associated with bradycardia and oxygen desaturation. The cessation of breathing lasted $90 \mathrm{~s}$, with one or at most two gasps; it was followed by two shorter apnea events.

\subsection{Additional examples}

We show three other examples of detected apnea events. The event in figure 4(a) lasted about $60 \mathrm{~s}$, with one apparent gasp or movement artifact in the middle. In this case, the NICU monitor worked properly. The measured respiration rate (blue circle-line) began to decrease a few seconds after the cessation of breathing, and dropped to 0 at $t=-2 \mathrm{~s}$; at that time the monitor signaled an apnea alarm. The large fluctuations in CI starting at $t \sim 40 \mathrm{~s}$ are most likely the result of NICU personnel stimulating the baby.

In figure 4(b), the apnea started at $t=-37 \mathrm{~s}$ and lasted for $74 \mathrm{~s}$. However, the monitor failed to detect this event. The reported respiration rate fell somewhat, but did not reach the threshold, then it increased to 105 breaths per minute at $t=-9 \mathrm{~s}$. Then the measured 

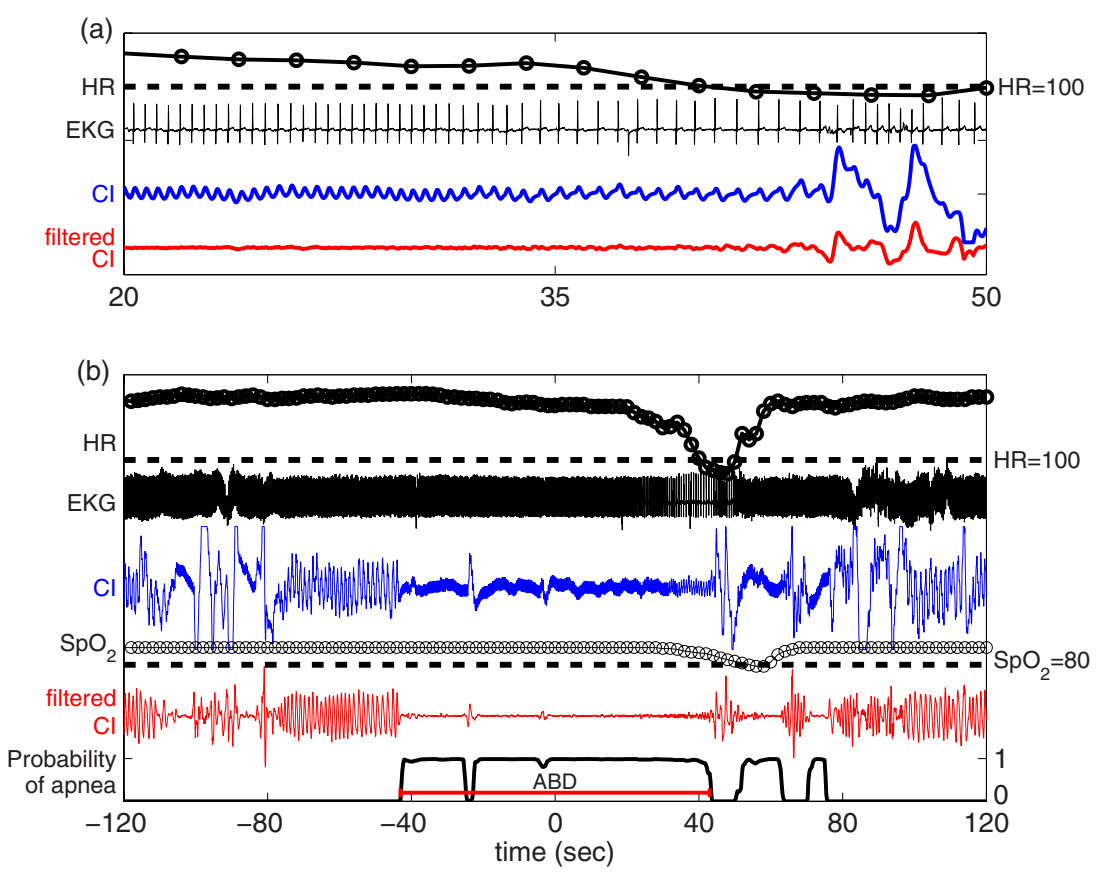

Figure 3. (a) $30 \mathrm{~s}$ of filtered CI (red) signal on an expanded scale, together with original CI, EKG and heart rate. (b) 4 min of filtered CI signal on a compressed scale, and the probability of apnea (black). The detected ABD event (apnea accompanied by bradycardia and oxygen desaturation) is marked (red bar).

respiration rate tracked the heart rate until $t=40 \mathrm{~s}$ (blue circles overlap black circles in figure 4(b)).

Figure 4(c) shows a periodic breathing event, or periodic apnea, in which the baby had a cycle with period about 15-18 s. In this case the CI signal is weak, and the cardiac artifact is large, so it takes a careful eye to see the periodic apneas in the original CI signal. On the other hand, it is very plain in the filtered signal. Further processing, such as computing a spectrogram of the probability of apnea curve, could provide a detector for periodic breathing.

\subsection{Validation of the new method for detection of central neonatal apnea}

The new algorithm was meticulously validated by comparing its output with analysis of signals by three expert clinicians, and parameters in the algorithm were adjusted to optimize agreement. Validation involved three separate analyses.

1. From the records of five infants selected for frequent episodes of apnea, we selected at random 237 episodes of coexisting bradycardia and $\mathrm{O}_{2}$ desaturation $(\mathrm{HR}<100 \mathrm{bpm}$, $\mathrm{SpO}_{2}<80 \%$ ). For each episode, we calculated the probability of apnea from analysis of the filtered CI signal, and three expert clinicians gave opinions on whether the episode was one of neonatal apnea. The clinicians reached consensus agreement on 234 of the 237 events. The new analysis of the filtered CI agreed with the clinicians in 212 cases out of those 234 (91\% accuracy). Validation data are shown in table 1.

2. We used the entire data set to evaluate the rates of false-positive and false-negative episodes of apnea detected by the new algorithms. To determine the false-positive rate, 

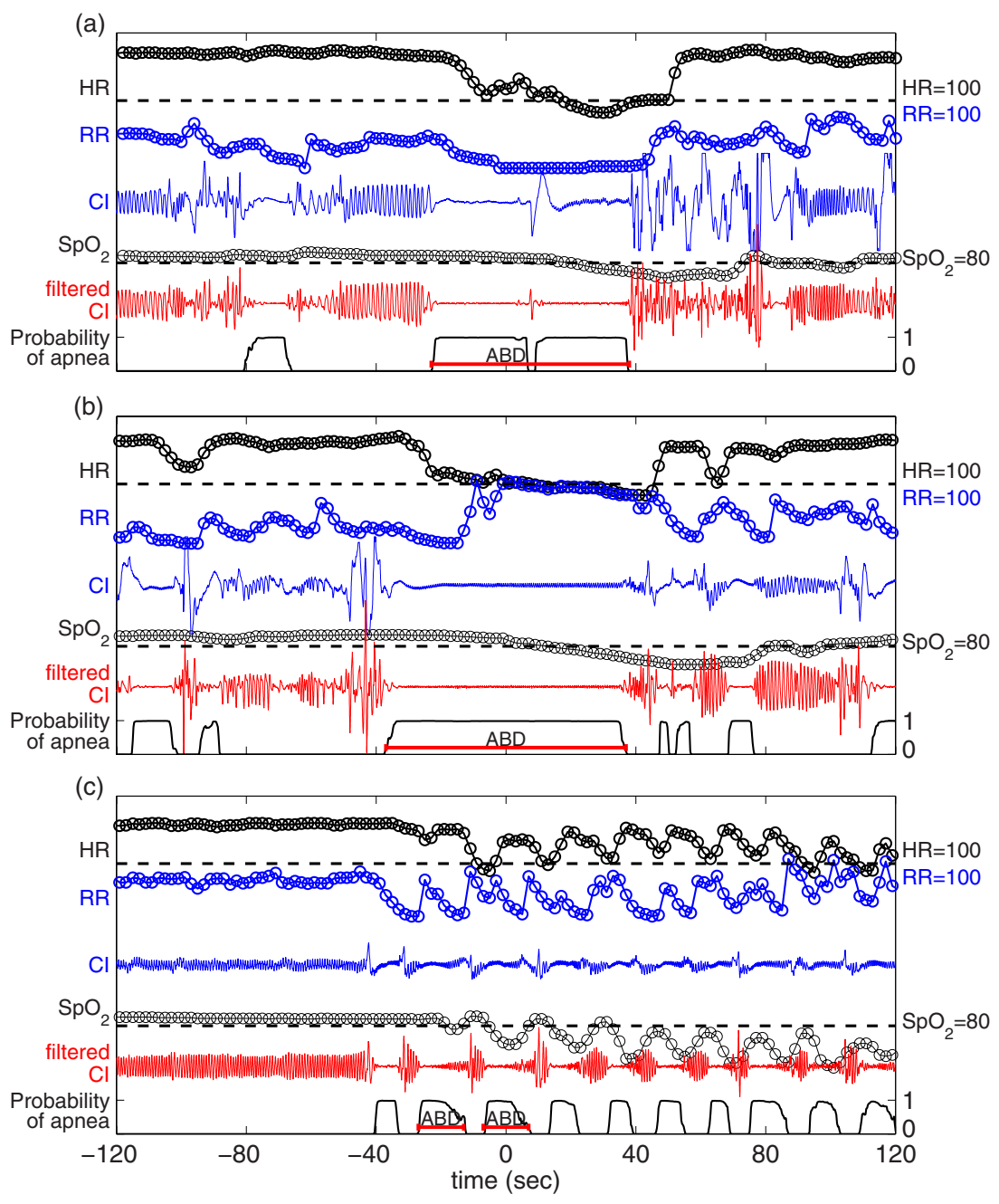

Figure 4. (a) An apnea event detected by the monitor. The respiration rate falls to 0.1 breaths per minute, and then jumps to zero. (b) An apnea event not detected by the monitor. The measured respiration rate equals the heart rate during the apnea. (b) Periodic breathing. The monitor shows cyclic rise and fall of respiration rate, while the new apnea detector shows periodic cessation of breathing.

Table 1. Validation result for data of five infants selected for frequent apneas.

\begin{tabular}{llrc}
\hline & \multicolumn{2}{c}{ Experts } \\
\cline { 3 - 4 } & & Apnea & Not apnea \\
\hline \multirow{2}{*}{ Computer } & Apnea & 132 & 7 \\
& Not apnea & 9 & 86 \\
\hline
\end{tabular}

we selected at random 100 episodes detected as apnea that lasted $30 \mathrm{~s}$ or more and were accompanied by bradycardia and $\mathrm{O}_{2}$ desaturation ('ABD-30' events). Three clinicians found that 95 of these represented episodes of central neonatal apnea, and the other five were false positives ( $5 \%$ false-positive rate). (In addition, for six of the 95 events, the 

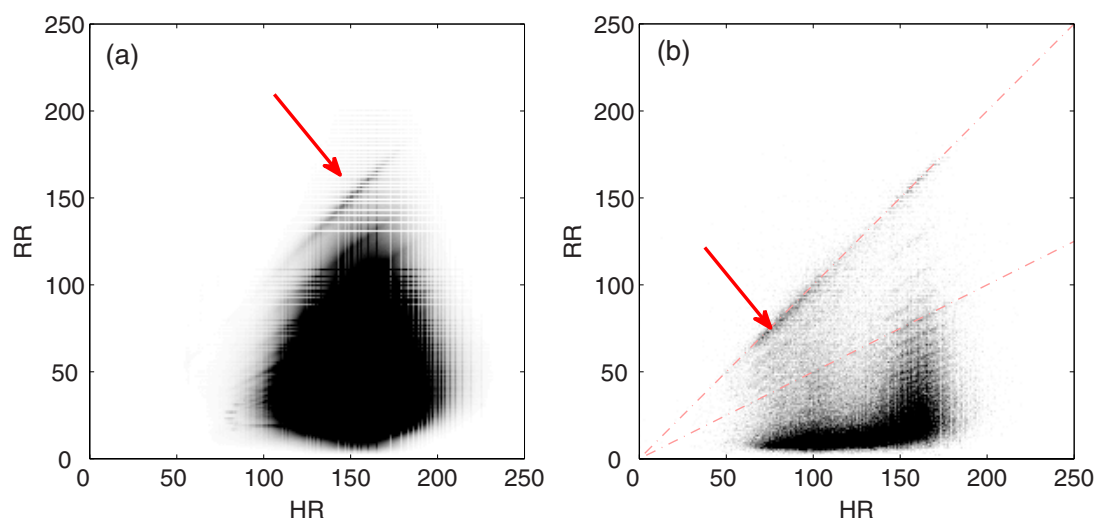

Figure 5. (a) Histograms of respiratory rate and heart rate in the entire data set, and (b) in apnea, bradycardia and $\mathrm{O}_{2}$ desaturation episodes lasting $30 \mathrm{~s}$ or more (ABD-30 events). The arrows point to a ridge at which the monitor records a respiration rate equal to the heart rate. Most of these are episodes of central apnea that were not detected by the monitor. About $14 \%$ of ABD-30 events are undetected because of the cardiac artifact.

clinicians concluded that the events were real apneas, but that they had lasted somewhat less than $30 \mathrm{~s}$; i.e. the algorithm overestimated the duration of the apnea, typically by just a few seconds. If we include such cases in the 'false positive' category, then the total false-positive rate is $11 \%$.)

3. To determine the false-negative rate, we randomly selected 114 apnea alarm episodes generated by the current monitoring system. After inspection of the monitor data, three expert clinicians concluded that 74 were false alarms and 40 were true alarms. Therefore, $65 \%$ of the monitors' apnea alarms were false. The new algorithms detected apnea in 39 of the 40 true alarm events, giving a false-negative rate of $2.5 \%$. The new algorithms also detected apnea in 43 of the 74 false alarm episodes, thereby giving a false-alarm rate of $37 \%$, a substantial improvement over the monitors. In every such case, the CI signal had very low amplitude. Since this is a common cause of apnea alarms from conventional monitors, this validation is biased toward unusually low amplitude CI signals. All apnea detection schemes are of limited value when the CI signal is flawed in this way.

\subsection{Application in the UVa database}

The availability of a quantitative measure of neonatal apnea allows examination of important research questions in the area. We have detected apneas using this method for our large database of monitor data for 1100 UVa NICU admissions since 1/2009.

3.4.1. Incidence of anomalous detection of respiratory rate $=$ heart rate and missed apnea alarms. Inspection of the monitor data in figure 1 suggests that a histogram of respiratory rate as a function of heart rate would show accentuation along the line of identity. Figure 5 shows this histogram for the entire data set (figure 5(a)) and for the episodes of apnea of $30 \mathrm{~s}$ or longer duration that were accompanied by bradycardia and $\mathrm{O}_{2}$ desaturation (ABD-30 events, panel B). The arrows indicate the line of identity, on which lie episodes of apnea in which the respiratory rate was erroneously detected to be the heart rate. In both panels, rates from 60 per 
minute to 160 per minute are represented. This is consistent with figure 1 , where the heartbeat was interpreted by the monitor as breathing from the beginning of apnea, at which time the heart rate was still normal.

We provide two measures of the incidence of such events: (1) In what fraction of the total time that babies are monitored does the monitor misinterpret heartbeats as respiration? (2) In what fraction of 'extreme' apnea events does the monitor misinterpret heartbeats as respiration?.

To answer the first question, we found that the proportion of data points under the line of identity in figure 5(a) (when HR and RR are identical within 5\%) was 0.0023 , suggesting that confusion of heart rate with respiratory rate by the monitors occurs $0.23 \%$ of the total time that the babies are monitored. That sounds like a small number. It is small because (happily) most of the time, the babies are breathing regularly, and the impedance changes due to respiration dominate over the impedance changes due to the cardiac artifact. However, when we examine apnea events, we see cause for concern.

To address the second question, we define an ABD-30 event as a central apnea with accompanying bradycardia and desaturation, in which the weighted apnea duration (see appendix) was at least $30 \mathrm{~s}$. Such events are called 'extreme' by Ramanathan et al (2001). In our 45-bed NICU, over 804 days we observed 7118 ABD-30 events (on average 1.6 events per day in 45 beds). Of those 7118 events, there were 1087 events in which the monitor-reported respiratory rate was close to the heart rate during at least part of the apnea. Of those cases, there were 1011 events in which the monitor did not give any apnea alarm because of confusing the heart rate with the respiratory rate. Thus, in 14\% of the ABD-30 events, the monitor failed to give an apnea alarm because of the cardiac artifact in CI signal. ${ }^{7}$

\subsection{Application to individual patients}

Figure 6 shows a NICU event map of an infant who died of necrotizing enterocolitis. The horizontal axis is NICU stay in days. The left vertical axis labels various events or treatments, and the white areas in the figure show when these treatments occurred. For example, the infant was given supplemental $\mathrm{O}_{2}$ in days 18 and 19, and again in the 40th day. NCPAP is nasal continuous positive airway pressure; HFNC and LFNC, high- and low-flow nasal cannula; $\mathrm{ABD}>30$ and $>10$, central apneas with bradycardia and $\mathrm{O}_{2}$ desaturations in which the apnea lasted more than 30 or more than $10 \mathrm{~s} ; A>20$, apneas lasting $>20 \mathrm{~s}$; Nurse AB, apnea and bradycardia nursing sheet entry.

The green line, referring to the right-hand axis, shows the number of ABD-30 events in the past $48 \mathrm{~h}$. For example, at some times in the 25th day, there had been more than five ABD-30 events in the past $48 \mathrm{~h}$. The red curve (also referring to the right-hand axis) is the HeRO score (Griffin and Moorman 2001, Griffin et al 2003, 2004, 2005a, 2005b, 2007, Lake et al 2003, Moorman et al 2006), which is a measure of heart rate variability that was developed to give early warning of sepsis. For example, in the 25th day, the HeRO score indicated that this infant was more than twice as likely as the average infant to have a sepsis event in the next $24 \mathrm{~h}$. A blood culture was drawn for suspicion of sepsis and seven days of antibiotics were given, though the culture was found to be negative.

7 The observant reader notices that there are stripe patterns in figure 5(b) along the slopes of rational numbers $(4 / 4$, $4 / 5,4 / 6,4 / 7,4 / 8$ and $4 / 9)$. As discussed above, the $4 / 4$ line (HR = RR, upper red dashed-dotted line) is due to the cardiac artifact in the CI signal. The $4 / 8$ line (lower red dashed-dotted line) is interesting. Most of those cases are false-positive reports of apnea, and this line shows that the true respiration rate and heart rate are sometimes locked in a 1:2 ratio. All other stripes in figure 5(b) are artifacts created by the monitor, which erroneously reports integer ratios of RR to HR when the CI signal is very weak. 


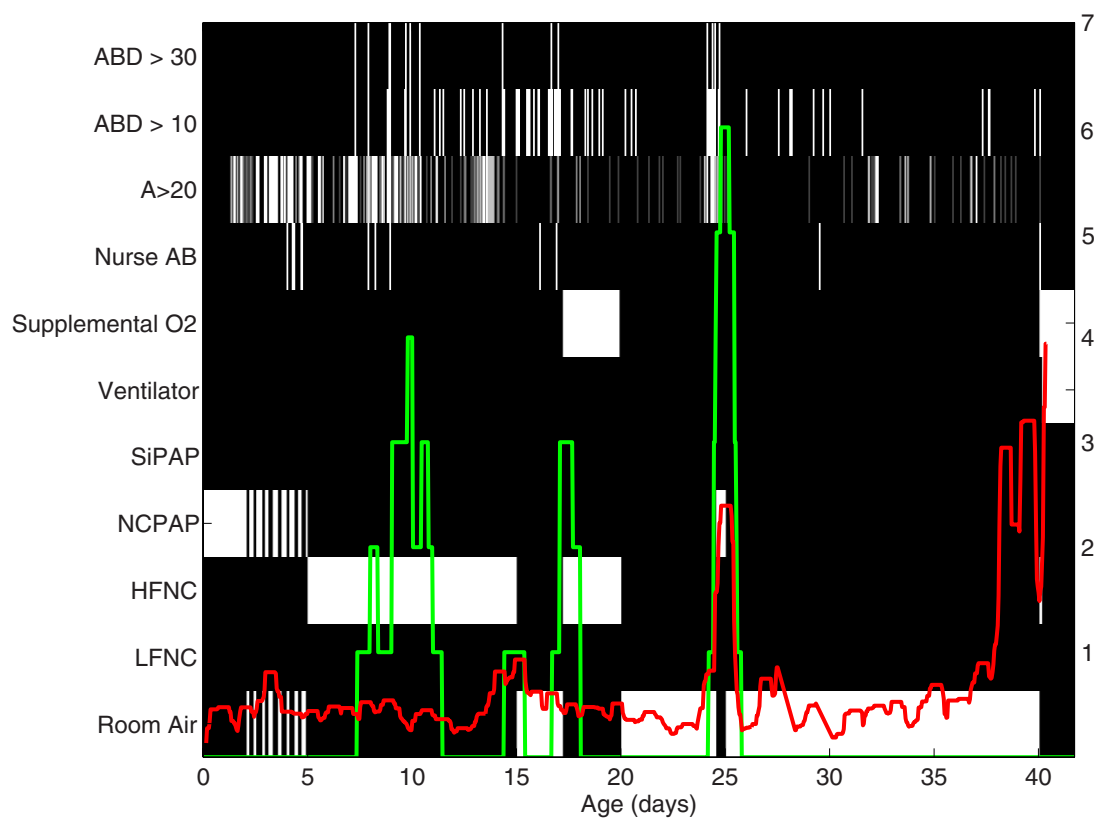

Figure 6. NICU event map for a single infant.

The primary points made by this figure are: (a) automated detection shows many more apneas than nurses' records show; (b) NICU personnel are responding to apneas by increasing the ventilatory support, but automated detection might lead to improved response; (c) sometimes the apnea rate is correlated with the HeRO score and sometimes it is not.

\section{Discussion}

Monitor algorithms sometimes mistake the cyclic impedance changes of cardiac filling and emptying as being due to respiration. We developed a novel filter based on resampling the CI signal based on the time between heartbeats, and used Fourier techniques to produce a filtered CI signal with the cardiac artifact removed. Overall, the incidence of central neonatal apnea that is unreported because of this phenomenon is significant ( $\sim 14 \%$ of ABD-30 events), and the missed episodes tend to be severe, with long duration and marked bradycardia.

More generally, an accurate and automated detector of central neonatal apnea should prove a useful tool in neonatal apnea studies. In 2006, an expert panel summarized the state of the art of neonatal apnea as inadequate in a number of respects (Finer et al 2006), beginning with an insufficient understanding of how to define apnea, let alone how to diagnose and treat it. They went on to review a number of hypotheses that need to be tested, such as the role of gastroesophageal reflux, and the effect of apnea on neurodevelopmental outcome, and the role of methylxanthine therapy. Such an algorithmic tool as we describe here should be useful for the rigorous quantification of the number and character of apnea episodes necessary to undertake these studies.

In addition, it will be interesting to see if bedside implementation of automated apnea detection alters practice. Our group recently participated in the HeRO trial, which showed that display of an algorithm based on heart rate that gives the fold-increase in risk of sepsis in the 
next $24 \mathrm{~h}$ reduced VLBW mortality by more than $20 \%$ (Moorman et al 2011a). It is likely that a similar display of the results of an apnea detector algorithm might also change clinical management in a beneficial way, or add to the existing sepsis detection algorithms (Moorman et al 2011b). In particular, it will be interesting to revisit the practice of waiting eight days after the last apnea episode prior to discharge (Darnall et al 1997). Such study may lead to better justification of this eight-day-countdown rule, or may lead to modification of the rule.

\subsection{Limitations}

The method cannot recognize obstructive apnea, which is often accompanied by struggling motions of the infant. As presently used, the method requires that the CI and EKG signals must have sufficient quality continuously over a 16 min period. Motional and other artifacts sometimes make either of these signals uninterpretable by human or machine. In addition, there are many cases in which the CI signal is of too low amplitude for reliable analysis. Also, at present, the detector is set up for retrospective use, not yet real-time use.

One other point should be mentioned. Some clinicians claim that apnea events themselves are not clinically important, unless there is an associated bradycardia or desaturation; therefore improved apnea detection by itself is not very important. We do not accept this claim. (a) The claim that apnea with no bradycardia or desaturation is clinically unimportant is an unproved hypothesis, which is not accepted for apneas of 20s or more (Finer et al 2006). (b) Even if it were true, an improved real-time apnea detection system could be combined with an automated system for stimulating the baby, and thereby preventing the subsequent bradycardias and desaturations which are more likely to be associated with morbidity. (c) Bradycardia and desaturation alarms are set at selected thresholds; we have seen many cases of long apneas in which the heart rate and oxygen saturation dropped substantially for long periods of time, but did not reach the thresholds to set off the alarms. This new system is providing better retrospective information than has previously been available, and we plan to adapt it to an accurate real-time early warning system.

\section{Conclusions}

We developed a new detection algorithm for apnea of prematurity. We substantially improve the apnea detection by removing the cardiac artifact from the CI signal, which cause false detection of apnea in the current generation of monitor. The validation with clinical experts shows more than $90 \%$ accuracy of the method.

\section{Acknowledgments}

This work was funded by NIH / NICHD GO grant 1 1RC2HD064488-02.

\section{Appendix}

\section{A.1. Filtering and scaling the signal}

The algorithm uses the three-lead EKG signals and the CI signal. From each lead of the EKG, QRS complexes are detected and $R$ times are recorded (Pan and Tompkins 1985). From the three lists of $R$ times, the most reliable one (the one that has the fewest missing beats) is chosen. Then for each RR interval, the $\mathrm{CI}$ is resampled by interpolation at 30 equally spaced intervals. 


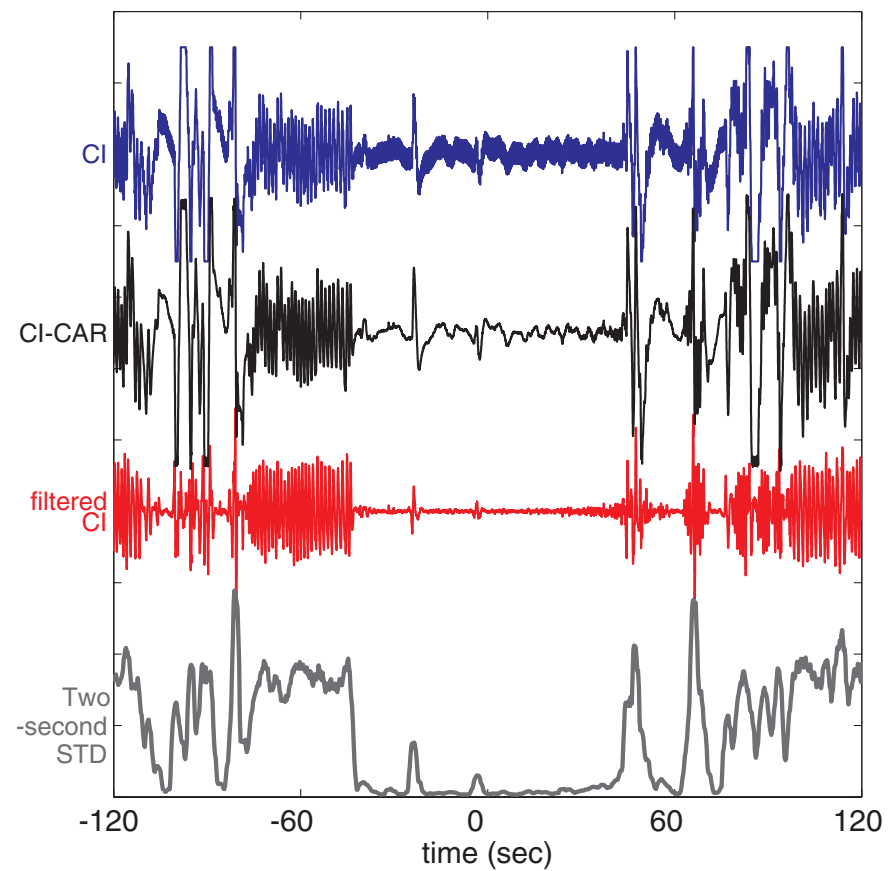

Figure A1. The same event as figure 3. Chest impedance (CI, blue), Chest Impedance-Cardiac Artifact Removed (CI-CAR, black), filtered chest impedance (FCI, red), and 2 s standard deviation (gray).

At this point, the Fourier transform looks like that shown in figure 2(b), the bands near integer frequencies can be removed, and the inverse transform can be computed, giving a signal with cardiac artifact removed. In practice, these three steps are combined into a single step using Butterworth bandstop filters. The result is then resampled at 60 points per second to obtain a filtered CI signal as a function of time. The name of this signal is Chest ImpedanceCardiac Artifact Removed (CI-CAR). An example is shown in figure A1.

This CI-CAR retains low frequency artifacts. Those are removed by a high-pass filter with threshold $0.4 \mathrm{~Hz}$. It is obvious that when this signal is silent, the infant is undergoing a central apnea event. But how silent is silent? The average amplitude of the original CI signal varies over a large range on time scales of minutes. Therefore we adjust the magnitude of the filtered signal dividing it by an envelope function which is computed by applying a low-pass filter with an extremely low thresholding frequency $(0.0025 \mathrm{~Hz})$ filter to the original CI signal. An example of this envelope function is shown in figure A2. This process can be called 'renormalization' of the filtered CI signal. The final result of the twice-filtered and renormalized CI is called the filtered CI (FCI). All further analysis is done with this signal.

\section{A.2. Apnea recognition}

We transform this filtered CI into a probability of apnea versus time. First we compute a running standard deviation $\sigma(t)$ of the scaled signal; we compute the standard deviation every quarter second for a centered $2 \mathrm{~s}$ interval (figure A1, same events as figure 3 ).

From that running standard deviation, we obtain an estimated probability of apnea using a transformation function that was obtained using two steps: (1) a preliminary formula was 


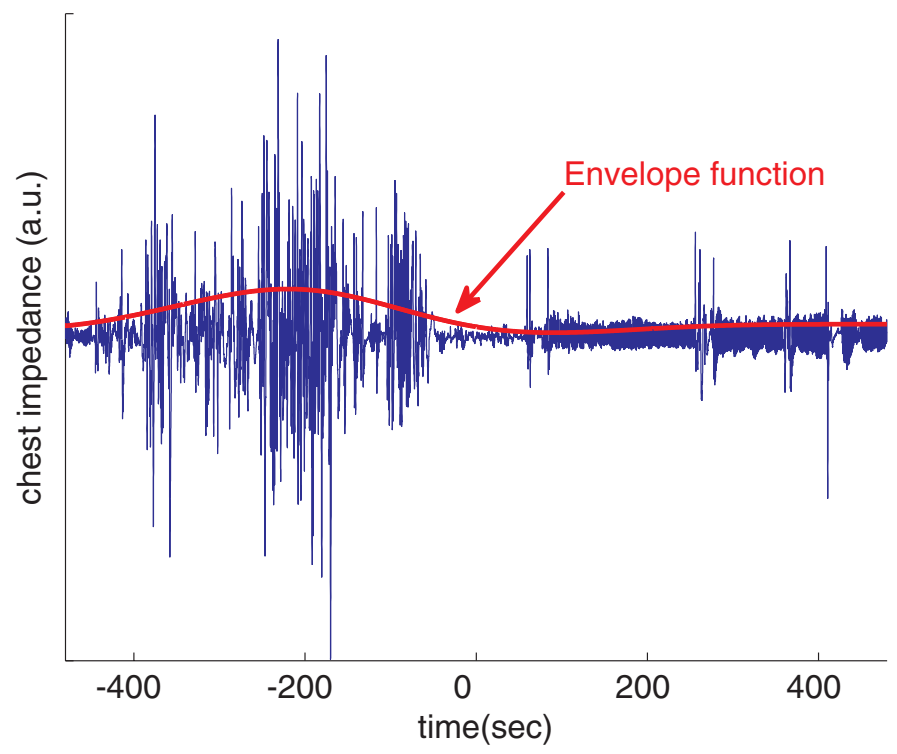

Figure A2. A very-low-pass filter (with threshold 1/400 Hz) provides an envelope function.
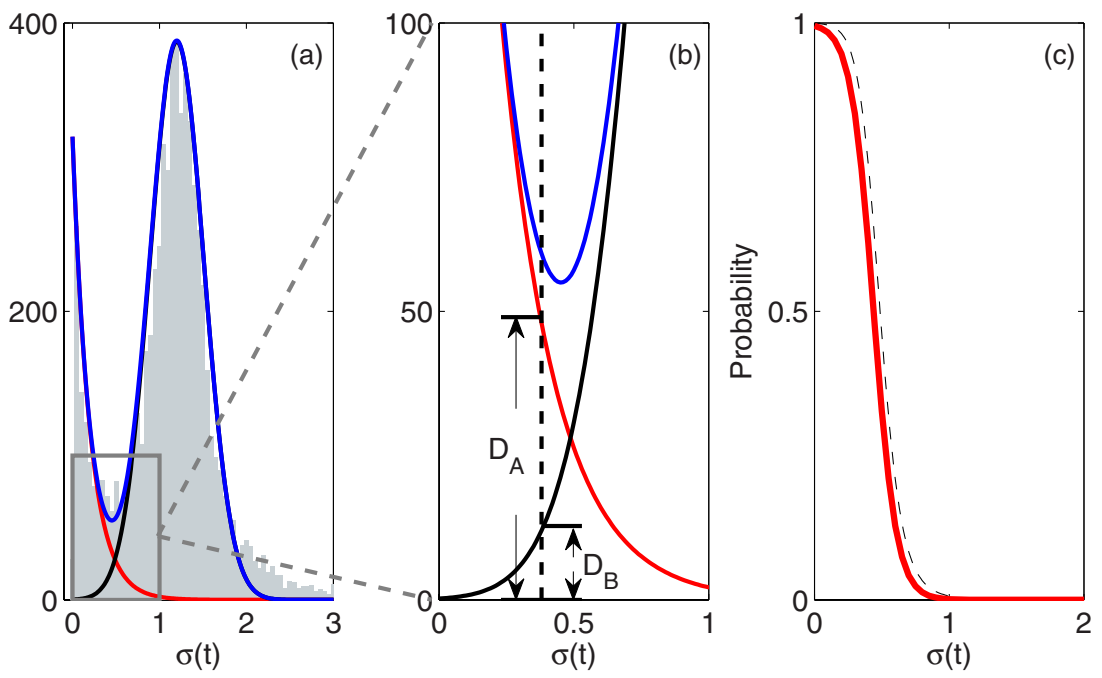

Figure A3. (a) Histogram of $2 \mathrm{~s}$ variance $(\sigma)$ of filtered chest impedance for a $4 \mathrm{~h}$ period in an infant with many apneas. The histogram is separated into two distributions. (b) Expanded view. The probability of apnea is given by $P(\sigma)=D_{A}(\sigma) /\left[D_{A}(\sigma)+D_{B}(\sigma)\right]$. (c) (Blue dashed line) the inferred probability of apnea versus $\sigma$ for the one very apneic infant. (Red solid line) the final choice of $P(\sigma)$ optimized as a result of the validation process after studying hundreds of apnea events in many infants.

obtained through study of a very apneic infant; (2) parameters in the formula were adjusted by a meticulous validation process in which three experts distinguished apnea from regular breathing for about 500 recorded events.

A histogram of values of $\sigma$ for one very apneic infant is shown in figure A3. We interpret this histogram as a statistical mixture of two distributions: $D_{A}(\sigma)$, which corresponds to apnea, 
and $D_{B}(\sigma)$, which corresponds to breathing. The total histogram is the sum of these two functions. The probability of apnea is given by $P=D_{A}(\sigma) /\left[D_{A}(\sigma)+D_{B}(\sigma)\right]$.

For convenience, the probability of apnea estimated in that way can be fit to a single function, $P_{\mathrm{fit}}(\sigma)=1 /[1+\exp [b(\sigma-a)]$ (called a Fermi function), with appropriate values for $a$ and $b$. Finally, the parameters $a$ and $b$ were adjusted by comparing the output of the formula with expert analysis of hundreds of apnea events in the NICU at University of Virginia ( $a=$ $0.44, b=12$ ). There is usually better than $90 \%$ agreement between the new apnea detection algorithm and the expert analysis. Details and results of the validation were given in the text. The strength of this approach is that we assign intermediate probabilities to signal values falling near the boundary between breathing and apnea.

\section{A.3. Apnea measures}

To obtain objective and quantitative measures of the frequency and severity of apnea events for each infant, we applied the following procedure. (1) Define the 'weighted apnea duration' (WAD) as the area under the probability of apnea curve $P(t)$, beginning when that probability increases through 0.1 , and ending when it decreases through 0.1. (2) Ignore all events with WAD less than $2 \mathrm{~s}$. (3) Ignore all events having WAD less than $5 \mathrm{~s}$ unless the event is within $5 \mathrm{~s}$ of another event. (4) Combine events if they are separated by less than $3 \mathrm{~s}$. For example in figure A1, we identify three events. The long apnea event begins at $t \sim-40 \mathrm{~s}$. At $t \sim-23 \mathrm{~s}$ there is an apparent gasp, and possibly another at $t \sim-3 \mathrm{~s}$. This apnea event ends at $t \sim 43 \mathrm{~s}$. We consider it to be a single event rather than two events because the single gasp interrupted it for less than $3 \mathrm{~s}$. The total duration of the event is $84 \mathrm{~s}$. WAD (area under curve) is $82 \mathrm{~s}$. The long event is followed by two short events, one of duration $11 \mathrm{~s}$ starting at $\mathrm{t} \sim 70 \mathrm{~s}$, and the second having duration about $6 \mathrm{~s}$. These two events are hard to recognize in the original CI curve, but they are plainly visible in the CI-CAR and FCI curves (figure A1). Note that during these latter events, the CI-CAR has some fluctuations, but they have no similarity to the regular breathing signals prior to $t \sim-50$.

\section{A.4. Definition of $A B D$ events}

We defined an ABD- $n$ event as an apnea in which the WAD is at least $n$ seconds, and there is an associated bradycardia (heart rate $<100 \mathrm{bpm})$ and desaturation $\left(\mathrm{SpO}_{2}<80 \%\right)$. Below we give a precise definition of 'associated': How long can the interval between these events be so that we will agree that the bradycardia and the desaturation are associated with (presumably caused by) the apnea?

First we state our rules, and then we explain how they were obtained. Define

$t_{A, i}=$ time of beginning of apnea (when the (black) probability of apnea curve increases through 0.1)

$t_{A, f}=$ time of end of apnea (when the (black) probability of apnea curve decreases through $0.1)$

$t_{B}=$ time of Bradycardia (when the average heart rate reported by the monitor falls through $100 \mathrm{bpm})$

$t_{D}=$ time of Desaturation (when $\mathrm{SpO}_{2}$ falls through 80\%).

$T_{B}=t_{B}-t_{A, i}=$ time interval from beginning of apnea to bradycardia

$T_{D}=t_{D}-t_{A, i}=$ time interval from beginning of apnea to desaturation

$\tau_{B}=t_{B}-t_{A, i}=$ time interval from end of apnea to bradycardia

$\tau_{D}=t_{D}-t_{A, i}=$ time interval from end of apnea to desaturation 


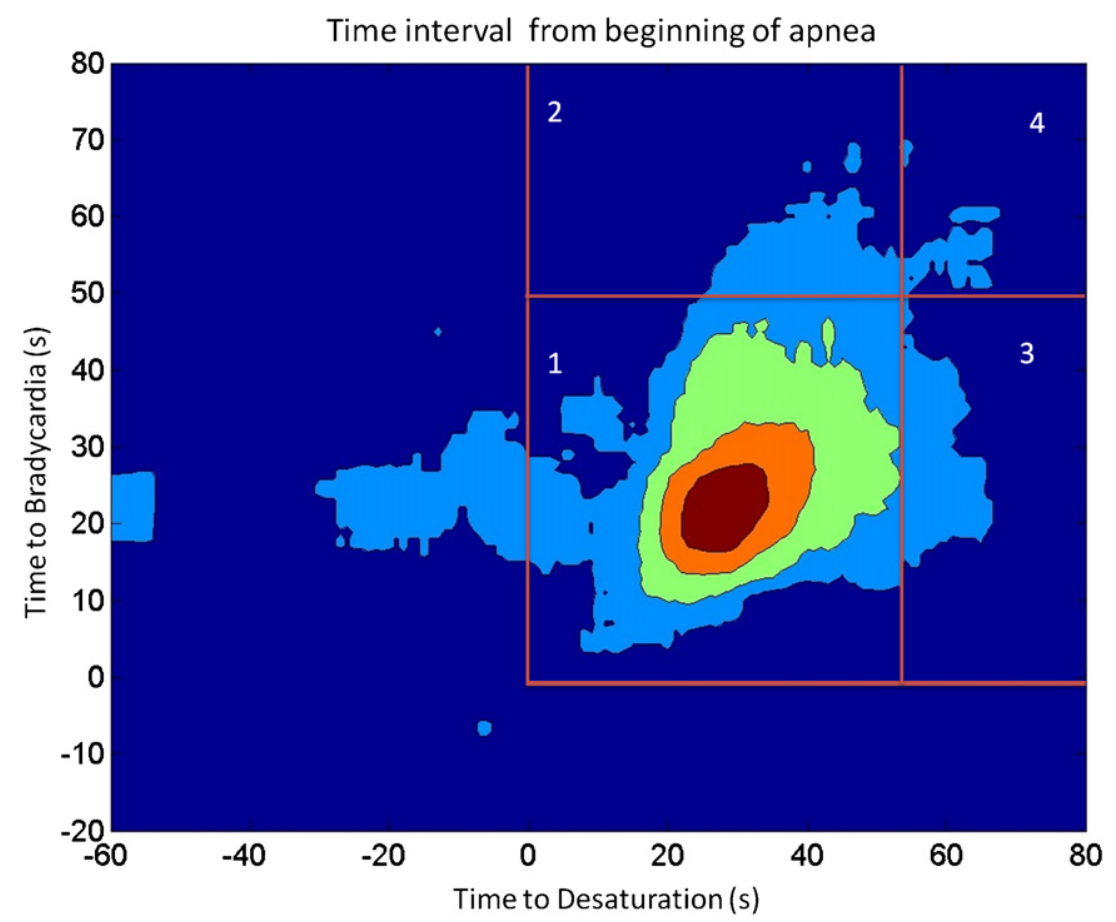

Figure A4. Histogram of joint distribution of times from beginning of apnea to bradycardia and to desaturation. Colors indicate pentiles of the distribution. Every event falling into box 1 is an ABD event. Any event falling into box 2 is an AD event (i.e. the desaturation is associated but the bradycardia is not), unless that event lies below the horizontal line in figure A5, in which case it is also an $\mathrm{ABD}$ event. Any event falling into box 3 is an $\mathrm{AB}$ event, unless that event lies to the left of the vertical line in figure A5, in which case it is again an ABD. Events falling into box 4 are presumed to be apneas with no associated bradycardia or desaturation, unless that event lies in the appropriate region of figure A5.

The rules we chose are

$$
\text { IF } T_{B}>0 \quad \text { AND } \quad\left[T_{B}<50 \mathrm{~s} \quad \text { OR } \quad \tau_{B}<25 \mathrm{~s}\right]
$$

then the Apnea and Bradycardia are related. (We call it an AB- $n$ event, with $n$ the Weighted Apnea Duration.)

$$
\text { (AD) } \quad \text { IF } T_{D}>0 \quad \text { AND } \quad\left[T_{D}<55 \mathrm{~s} \quad \text { OR } \tau_{D}<38 \mathrm{~s}\right]
$$

then the Apnea and Desaturation are related (it is an AD- $n$ event). If both of the conditions $\mathrm{AB}$ and $\mathrm{AD}$ hold, it is an $\mathrm{ABD}$ event.

We obtained these rules by the following process. We began by studying five very apneic infants who were not on ventilators. The computer identified 2664 events in which a bradycardia and a desaturation occurred within a 4 min window. Then for each such event, the monitor output was examined by one or more clinicians, and (particularly looking at the CI signal and the monitor-derived respiration rate) the clinicians identified 1897 apnea events, 932 of which were judged to be central apneas. They then manually recorded the times of the beginning and the end of the apnea. Times of bradycardia, $t_{B}$ and desaturation $t_{D}$ were recorded electronically in our database. This information was used to generate the joint histograms of time differences shown in figures A4 and A5. 


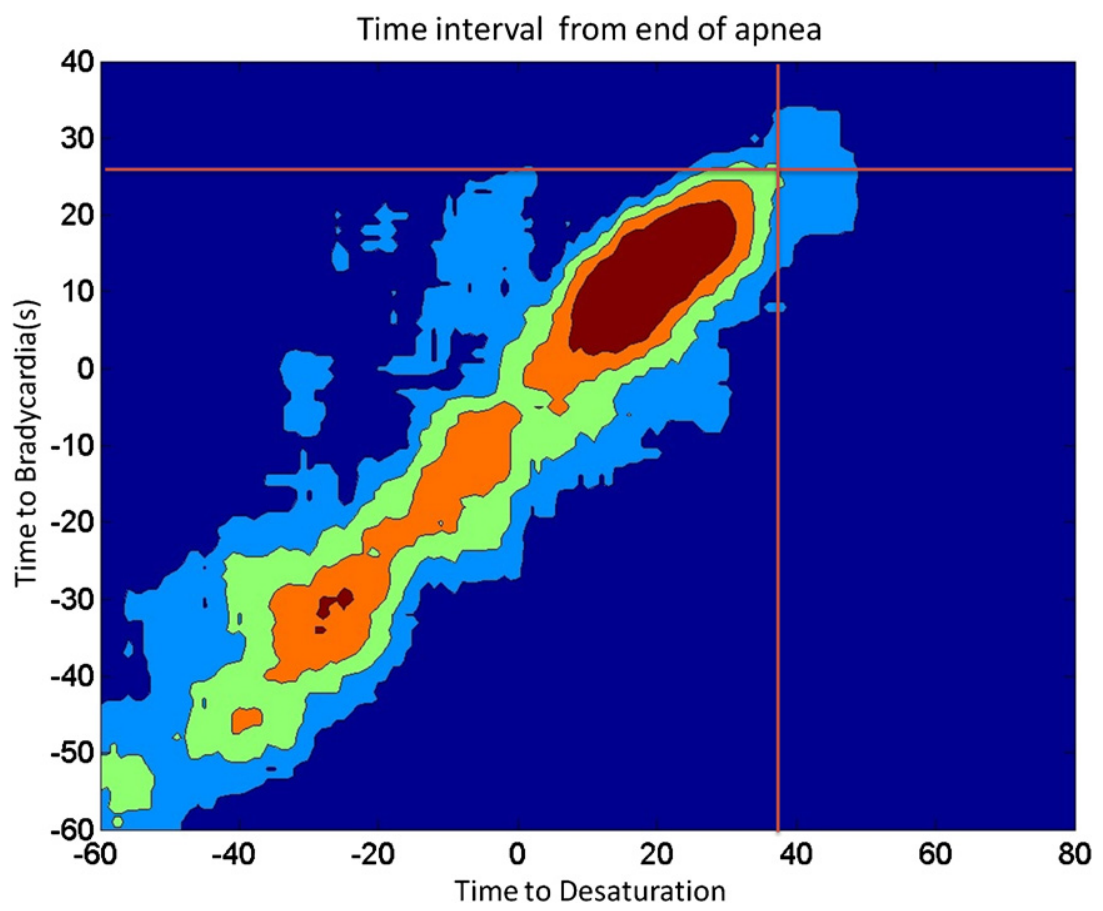

Figure A5. Histogram of joint distribution of times from end of apnea to bradycardia and to desaturation. Colors indicate pentiles of the distribution. Any event in the lower left region of this figure is declared to be an ABD event provided that the said event meets the criteria in figure A4.

The first part of condition $\mathrm{AB}$ says that if the crossing of the bradycardia threshold occurred before the beginning of apnea, then it was not caused by the apnea. The second part says that the bradycardia must occur within a reasonable time after the beginning or within a reasonable time after the end of the apnea. The same logic was applied for defining AD events. The boundaries we selected are shown on these histograms. Each boundary $(50,55,25$ or $38 \mathrm{~s}$ ) is somewhat arbitrary, and could have been chosen to be smaller by a few seconds, or larger by at most $20 \mathrm{~s}$. The validity of the rules and the boundaries are supported also by the validation process discussed earlier, in which three experienced clinicians examined hundreds of events.

\section{References}

Darnall R A, Kattwinkel J, Nattie C and Robinson M 1997 Margin of safety for discharge after apnea in preterm infants Pediatrics $\mathbf{1 0 0} 795$

Finer N N, Higgins R, Kattwinkel J and Martin R J 2006 Summary proceedings from the apnea-of-prematurity group Pediatrics 117 S47

Griffin M P, Lake D E, Bissonette E A, Harrell F E, O'Shea T M and Moorman J R 2005a Heart rate characteristics: novel physiomarkers to predict neonatal infection and death Pediatrics 116 1070-4

Griffin M P, Lake D E and Moorman J R 2005b Heart rate characteristics and laboratory tests in neonatal sepsis Pediatrics 115 937-41

Griffin M P, Lake D E, O'Shea T M and Moorman J R 2007 Heart rate characteristics and clinical signs in neonatal sepsis Pediatr. Res. 61222 
Griffin M P and Moorman J R 2001 Toward the early diagnosis of neonatal sepsis and sepsis-like illness using novel heart rate analysis Pediatrics 107 97-104

Griffin M P, O'shea T M, Bissonette E A, Harrell F E, Lake D E and Moorman J R 2003 Abnormal heart rate characteristics preceding neonatal sepsis and sepsis-like illness Pediatr. Res. 53 920-6

Griffin M P, O'shea T M, Bissonette E A, Harrell F E, Lake D E and Moorman J R 2004 Abnormal heart rate characteristics are associated with neonatal mortality Pediatr. Res. 55782

Jong W 2000 Blood pressure variability in neonates: with a special focus on signal acquisition and signal processing Dissertation Technische Universiteit Eindhoven

Judell N H K 1983 US Patent No. 4,379,460 (issued 12 Apr. 1983)

Lake D E, Griffin M P and Moorman J R 2003 New mathematical thinking about fetal heart rate characteristics Pediatr. Res. 53 889-90

Moorman J R et al 2011a Mortality reduction by heart rate characteristic monitoring in very low birth weight neonates: a randomized trial J. Pediatr. $159900-6$

Moorman J R et al 2011b Cardiovascular oscillations at the bedside: early diagnosis of neonatal sepsis using heart rate characteristics monitoring Physiol. Meas. 321821

Moorman J R, Lake D E and Griffin M P 2006 Heart rate characteristics monitoring for neonatal sepsis IEEE Trans. Biomed. Eng. 53 126-32

Pan J and Tompkins W J 1985 A real-time QRS detection algorithm IEEE Trans. Biomed. Eng. 32 230-6

Poets CF 2010 Apnea of prematurity: what can observational studies tell us about pathophysiology? Sleep Med. 11 701-7

Ramanathan R et al 2001 Cardiorespiratory events recorded on home monitors JAMA 2852199

Wright J C and Triebel H M 1988 US Patent No. 4,781,201 (issued 1 Nov. 1988) 